\title{
La communication de changement : vers une heuristique de l'induction
}

\section{Françoise Bernard}

\section{(2) OpenEdition}

12 Journals

Édition électronique

URL : http://journals.openedition.org/communicationorganisation/2001

DOI : 10.4000/communicationorganisation.2001

ISSN : $1775-3546$

Éditeur

Presses universitaires de Bordeaux

\section{Édition imprimée}

Date de publication : 1 novembre 1997

ISSN : 1168-5549

\section{Référence électronique}

Françoise Bernard, "La communication de changement : vers une heuristique de l'induction », Communication et organisation [En ligne], 12 | 1997, mis en ligne le 26 mars 2012, consulté le 20 avril 2019. URL : http://journals.openedition.org/communicationorganisation/2001 ; DOI : 10.4000/ communicationorganisation.2001

Ce document a été généré automatiquement le 20 avril 2019.

(c) Presses universitaires de Bordeaux 


\title{
La communication de changement: vers une heuristique de l'induction
}

\author{
Françoise Bernard
}

1 En micro-économie et en communication organisationnelle, la notion de changement demeure incontestablement une notion d'actualité. Cette actualité nécessite d'être replacée dans un contexte plus large qui révèle que le thème du changement organisationnel est bien antérieur aux années 80 . Le contexte à prendre en compte pour notre réflexion est de dimension macro-socioéconomique, c'est celui de l'histoire des révolutions industrielles. Cette histoire (Verley, 1997) montre que le changement est permanent. Il est fortement soudé aux évolutions technologiques, la dernière d'entre elles étant marquée par la généralisation des technologies informationnelles et intelligentes.

2 Par ailleurs, l'entreprise est dans son siècle, elle est affectée par le mouvement de l'histoire en général mais aussi plus spécifiquement par les mouvements sociaux qui ont apporté une contribution à la constitution du modèle patrimonial-contractuel français (Segrestin, 1996). Le changement organisationnel n'est en quelque sorte qu'une des micro figures de ce macro changement continu. Il suffit d'un retour en arrière pour établir le constat de la permanence du discours de changement. Par exemple, dès le début des années 60 , le thème de la réforme de l'entreprise était déjà présenté comme une nécessité (Bloch Lainé, 1963, Sudreau, 1975). Cependant dans ces années là et jusqu'à la fin des années 70 , il y avait une forme de réciprocité entre la réforme de l'entreprise et la réforme de la société par les conflits sociaux (Adam et Reynaud, 1978). Alors qu'aujourd'hui, l'économique semble guider l'ensemble des choix économiques et non économiques. Plus techniquement, tantôt le changement organisationnel est produit par l'introduction d'un nouveau modèle de production, tantôt par la généralisation et la consolidation de celui-ci, tantôt enfin par son déclin et par son remplacement par d'autres modèles. Autrement dit, à l'échelle des organisations le changement est régulier, y compris dans des phases de stabilité macro-économique. La phase des trente glorieuses, correspondant à la phase A d'expansion du quatrième cycle long de l'économie mondiale (Gilles, 1996), a été marquée par l'hégémonie du modèle taylorien-fordien, modèle qui a 
été généralisé en France dans les années 60. Il correspondait à un certain niveau technologique et culturel. À ce modèle est associé un modèle communicationnel descendant, linéaire, mécaniciste.

3 Ce modèle tend à être remplacé depuis une quinzaine d'années par un nouveau modèle, le modèle flexible toyotiste ou ohniste. Ce dernier correspond à un nouveau niveau technologique et culturel, celui de l'introduction massive dans les organisations des technologies intelligentes (robotisation, informatisation, télécommunications, etc.). C'est le mouvement de "reengineering» (Hammer et Champy, 1993) traduisible en langue française par les termes de reconfiguration, de reconception des processus de l'entreprise ${ }^{1}$, ou encore de recomposition organisationnelle. Les caractéristiques du modèle ohniste peuvent être résumées en dix points :

4 - le développement de la robotisation et des nouvelles technologies de l'information et de la communication (Bardelli, 1992)

5 - la maîtrise des flux et en particulier des flux d'information (Karcher, 1988)

6 - l'extension de la flexibilité (Cohendet et Llerena, 1989)

7 - la mise en place d'une coordination interentreprises en particulier avec les réseaux donneurs d'ordre-sous traitants (Brousseau, 1993 ; Baudry, 1995)

8 - la généralisation des normes, des procédures des référentiels

9 - la gestion en juste à temps et à flux tendus

10 - la qualité totale

11 - le temps réel et l'immatérialité des transferts (information, finances, etc.)

12 - les questions de la polyvalence et de la compétence des salariés

13 - une redéfinition de l'interface conception et réalisation du travail (Liu, 1988).

14 À ce modèle sont associées certaines pratiques communicationnelles : déverticalisation de la communication ou mise en place d'une communication horizontale et transversale, valorisation de la qualité de la communication informelle (Paraporanis, 1990), accroissement de la multicanalité (aux canaux traditionnels s'ajoutent les canaux électroniques), nouvelles formes de coopération internes et externes. La question est de savoir si ces nouvelles pratiques émergeantes font rupture avec le paradigme communicationnel dominant ou si elles ne sont que des adaptations de celui-ci. Cette question est étroitement reliée à une autre question: celle qui porte sur la nature néotaylorienne ou post taylorienne de ce nouveau modèle. Autrement dit le débat relatif à l'émergence ou non d'un changement de paradigme organisationnel est ouvert depuis le début des années 90. Pour certains auteurs, en particulier ceux de l'école française, il s'agirait plutôt d'une modification du fordisme-taylorisme (Wood, 1993, Linhardt, 1994), pour d'autres d'un nouveau paradigme, voire $\mathrm{d}$ "'un système qui va changer le monde » (Womarck, Jones, et Roos, 1992).

15 Cependant, au-delà de ce débat, il y a bien un discours ambiant sur la modernité de la reconfiguration organisationnelle en cours qui est associé au vieux présupposé évolutionniste du changement. Celui-ci nourrit une représentation déterministe de l'évolution technico-économique. En écho, toute pensée qui nuance l'approche déterministe est promptement qualifiée de passéiste et de corporatiste, c'est-à-dire disqualifiée. 
16 Dans ce cadre général, nous souhaitons défendre l'idée selon laquelle le changement lié au mouvement de reconfiguration organisationnelle qui occupe le devant de la scène, ne résume pas à lui tout seul la dynamique de changement des organisations. Nous mettrons en évidence d'autres logiques et d'autres pratiques de changement, par le bas, ordinaires, quotidiennes et permanentes. Autrement dit dans le grand flux des événements (Bateson, 1989), le changement prescrit n'est qu'une figure événementielle spécifique parmi bien d'autres qui pourraient tout aussi bien être nommées « changements ». Parmi ces autres figures événementielles, nous allons privilégier toutes les formes de micro coopération inventées, expérimentées à la base. Celles-ci permettent l'efficience de l'organisation prescrite en général mais aussi la mise en œuvre concrète du changement prescrit. Nous considérons donc que coexistent dans l'organisation différentes situations de changement caractérisées par différentes logiques de communication. Nous les avons classées en deux types:

17 - les situations de type 1, il s'agit des changement technico-organisationnels prescrits par le management

18 - les situations de type 2, il s'agit du changement au quotidien tel qu'il est co-produit et vécu par les acteurs en phase de changement prescrit et hors phase de changement prescrit.

19 D'un point de vue méthodologique, et à l'échelle de chaque organisation, la question du changement peut être saisie par le haut ou par le bas. Bien évidemment, changements par le haut et changements par le bas interagissent dans une dynamique générale du changement. Tout change ensemble ${ }^{2}$, le technique, le social et l'organisationnel. Cependant, nous pouvons noter une forme d'asynchronie entre changements par le haut et changements par le bas. Les premiers sont séquentiels et discontinus, les seconds sont discrets et continus.

20 Nous souhaitons démontrer que la question du changement ouvre des brèches communicationnelles :

21 - dans les situations de type 1, avec des rétroactions et des effets de cadre peu analysés

22 - dans les situations de type 2, avec l'émergence d'une communication largement informelle méconnue ou oubliée qui ne peut être saisie scientifiquement que par un élargissement des cadres de référence d'études en communication organisationnelle, par exemple avec la prise en compte du paradigme de l'induction.

\section{La communication managériale de changement : régulation et pilotage}

23 Les situations de type 1, correspondent aux changements par le haut. Il s'agit d'un changement poussé par le management en réponse aux évolutions des marchés et des technologies.

24 Ils correspondent à des moments présentés comme spécifiques et non ordinaires de la vie d'une organisation. Le changement prescrit est accompagné d'une production d'énoncés sur le changement. Dans ces situations, le changement est poussé par l'encadrement. Tantôt le changement est présenté comme un moment particulier, non ordinaire de la vie d'une organisation, tantôt il est présenté comme le nouvel élément structurant et désormais permanent lié à une économie mondialisée, un véritable trait culturel. La 
question que nous nous posons est de savoir si à un nouveau modèle de production est associé un renouvellement de la pensée et des pratiques de changement ainsi qu'un nouveau modèle communicationnel?

Sur la base des études de cas que nous avons effectuées, nous formulons l'hypothèse selon laquelle le modèle de changement à l'œuvre se fait selon les règles, au moins implicitement, du pilotage managérial classique. Le cadre dominant reste celui du centralisme, de la linéarité, celui du schéma canonique de la communication - émetteurrécepteur - (Moles, 1986). Il provoque de ce fait des réponses qui sont généralement traduites dans les termes très prévisibles de l'adaptation ou de la résistance.

\section{Circularité recommandée, linéarité implicite}

Choisir les termes de régulation et de pilotage pour désigner l'intervention managériale de changement nous situe de plain-pied dans le paradigme de la cybernétique et plus précisément dans la première cybernétique. Il en va de même lorsqu'on évoque la logique circulaire de certains processus. Nous allons examiner certaines formes et certaines limites de cette approche que l'on peut qualifier d'organiciste (Sfez, 1988). Pour cela nous allons distinguer deux périodes, la période d'implantation du changement puis la période d'expérimentation du nouveau modèle.

En ce qui concerne la première période, celle de l'implantation du changement, on note que le changement impulsé par les directions est souvent présenté comme un temps singulier de la vie des organisations. Cependant on note également qu'il est piloté selon les formes linéaires habituelles de la communication de non changement. En particulier, le quadrillage de l'action de lancement révèle une phobie entro-pique qui renvoie aux pratiques générales et linéaires de la communication managériale. Tout se passe comme si le hasard et le bruit donnaient lieu assez systématiquement à des tentatives de réduction fortes. Un tel constat révèle d'une part la fragilité, dans les pratiques, de l'intégration de la cybernétique et de la systémique et, d'autre part, une ignorance de fait de l'apport de la deuxième cybernétique.

Les présupposés et les pratiques du changement managérial demeurent donc, y compris en phase de changement, fondamentalement linéaires, même si ponctuellement on peut observer ça et là la mise en place de boucles de rétroaction sous forme de consultation, de concertation et de participation. Une telle logique circulaire de la communication reste globalement limitée à des séquences précises par exemple: de recueil de données, ou encore d'ajustement culturel avec une culture d'équipe ou une culture de métier spécifiques. Si la linéarité est dominante c'est aussi parce que le deuxième présupposé structurant est de nature béhavioriste. En l'occurrence, aux stimuli managériaux doivent répondre des adaptations de structure et d'acteurs.

Généralement, le pilotage comporte les phases suivantes :

en amont une conception centralisée du projet de changement, le contenu en étant défini par une équipe limitée composée d'acteurs stratégiques, d'experts et de décideurs,

31 - le processus du changement est préconçu de manière séquentielle, il est ensuite accompagné selon une logique descendante,

32 - la régulation de la réalisation du changement s'effectue sous forme alternée de négociation et de passage en force. Cependant le passage en force est généralement privilégié lorsqu'un obstacle majeur émerge. 
33 Au niveau des effets de cadre, la communication de changement poussée met l'accent sur la rationalisation par les normes et les procédures susceptibles d'assurer le succès du changement. Par exemple, dans un projet de mutualisation d'établissements socioéducatifs et sanitaires de la sécurité sociale, il s'est agi de définir des référentiels d'action susceptibles de rationaliser les activités et de renforcer le contrôle de gestion. Autre exemple, à France Telecom, il s'est agi dans certaines directions régionales, par l'organisation d'un forum des métiers, d'initier le personnel à des procédures de changement de métier qui accompagnent en fait les changements de structure dont on connait aujourd'hui la définition. Ces normes et procédures sont prépensées et préconçues, en tout cas au moins au niveau des méthodes, y compris si un processus de consultation est généralement mis en œuvre.

34 La normalisation par les procédures s'accélère depuis le début des années 90 (normes qualité, référentiels, etc.). Nous considérons même que la normalisation, dans l'acception de mise en norme, gagne le champ de l'intersubjectivité, comme

En ce qui concerne la deuxième période, celle de l'expérimentation du modèle de changement, on peut mesurer les effets communicationnels des évolutions liées au passage d'un modèle de production poussé par l'offre à un modèle de production tiré par la demande. Deux effets sont à noter :

361 - la nécessité de la mise en place de boucles de rétroaction, c'est-à-dire d'une logique de communication circulaire motivée par la contrainte de réactivité et d'anticipation se distinguant de la logique linéaire descendante du modèle précédent.

372 - La nécessité de favoriser un ensemble de micro-coopérations intra-équipe, interéquipes, inter-entreprises (Schieb, 1986) qui correspond aux mises en réseau, c'est-à-dire une logique non linéaire où l'imprévisibilité est davantage admise. Nous analyserons cet effet plus loin ${ }^{3}$.

Nous allons donc développer le premier effet celui de la circularité. Il y a bien passage du paradigme linéaire de la communication au paradigme circulaire. Cependant la circularité en question mérite une analyse. Dans ce but, il nous semble intéressant en effet de croiser les notions de circularité et de symétrie (Watzlawick, 1972). Dans le nouveau modèle circularité et symétrie fonctionnent simultanément à un certain niveau, celui de la gestion et de la coordination de la production, à d'autres niveaux la circularité est largement dominée par l'asymétrie. Parmi ces niveaux, on peut citer celui de la structure interentreprises. Il existe bien asymétrie et linéarité entre les entreprises sous traitantes qui sont dominées et les entreprises donneurs d'ordre qui sont dominantes. Celles-ci détiennent les informations stratégiques et fixent les règles du jeu. On peut aussi citer certaines interventions pour accroître la flexibilité où l'on mesure que les nous l'analysons plus loin. ressources humaines sont assimilées à des obstacles vers plus de flexibilité; la symétrie communicationnelle de production est parfois pour ce motif brutalement rompue (fermeture de site industriel, plan social, licenciement).

39 En résumé en période d'implantation ou en période d'expérimentation, on peut de la même façon, distinguer les phases de communication circulaire que l'on peut qualifier de conjoncturelles et d'opportunistes car elles sont à cycle court et des phases de linéarité que l'on peut qualifier de structurantes car elles sont à cycle long. 


\section{Le changement par réaction : une circularité méconnue}

40 En réponse à cette communication de changement poussée, les acteurs peuvent penser et réagir de différentes manières. Tout d'abord, on peut noter qu'au codage dans les messages d'émission officiels et formels du changement répondent des opérations de traduction et de surcodage dans les messages de réception informels. Le surcodage se fait différemment en fonction de différentes variables : la crédibilité de la source, l'évaluation stratégique des gains et des pertes, la qualité de la communication informelle entre niveaux hiérarchiques, la congruence hiérarchique (Bernard-Peyrolle, 1991), etc. Parmi l'ensemble des réactions, sont très souvent évoquées du côté managérial, les résistances au changement des salariés. Cette notion est une sorte de fourre tout dans lequel sont classées de nombreuses rétroacations qui ont pour particularité de révéler certaines dimensions oubliées du changement. Cette notion relève selon nous, du paradigme linéaire. D'une part, sur la ligne descendante du changement prescrit, la résistance serait en quelque sorte le dernier obstacle avant l'adaptation. D'autre part, la notion de résistance est connotée comme un refus de la modernité qui est supposée être incluse dans chaque changement. Dans la mesure où le changement est hanté par le présupposé positiviste du progrès, on comprend que la résistance au changement soit socialement dévalorisée.

Cette notion, dans la dynamique organisationnelle, nous semble être avantageusement remplaçable par la notion de boucles de rétroaction dans le processus continu de changement. À y regarder de près ces boucles de rétroaction proposent un élargissement du cadre d'interprétation du changement prescrit. Ainsi, à leur manière, les acteurs « résistants » ombrent les certitudes et les méthodes d'une certaine pensée économique. En particulier les résistances sont liées à une mise en cause de ce qui est très souvent présenté comme un déterminisme économique et technologique. Mieux les résistances sont parfois accompagnées de contre projets qui reposent sur d'autres rationalités que celles du changement prescrit. Enfin, les résistances exercent une fonction d'homéostasie qui révèlent les enjeux identitaires du précaire équilibre entre changement et permanence.

Nous venons d'examiner le changement par réaction, nous allons étudier le changement par proaction.

En conclusion, nous pouvons considérer qu'au niveau logique les situations de communication managériale de changement relèvent d'une logique de la déduction à deux niveaux :

- il s'agit bien de réguler « de l'extérieur » des processus en fonctions de règles explicites ou implicites,

45 - il s'agit bien d'interpréter la variété des comportements en terme de "réponse » et de décoder ces réponses à partir d'une grille d'intelligibilité implicite, la grille linéaire.

\section{La communication du changement vécu : non linéarité}

Les situations de type 2, correspondent au changement par le bas. Il s'agit d'un changement tiré par les acteurs. Il se traduit par des microactes qui correspondent aux initiatives des acteurs dans un certain contexte. Ce contexte peut être un contexte de non 
changement prescrit, mais aussi, de changement prescrit. Dans ce dernier cas il y a donc symétrie et complémentarité entre deux dynamiques de changement.

Plus généralement, en ce qui concerne les changements tirés, notre hypothèse revient à considérer qu'il y a une expérience du changement correspondant à la quotidienneté de la vie des organisations pour les individus au travail. Ceux-ci, y compris dans les phases où le cadre plus général évolue peu, sont amenés quotidiennement à résoudre des micro problèmes matériels (organisation, techniques, pannes, imprévus et contretemps de toute nature, etc.) ou immatériels (interactions, coopération, conflits, négociation etc.). La résolution de ces micro problèmes qui nécessite, pour certains d'entre eux, de mobiliser créativité, inventivité et bricolage, s'effectue dans le cadre de micro coopérations. Cellesci s'actualisent dans les réseaux informels constitués par chaque acteur, réseaux qui peuvent être stables ou éphémères. On peut ainsi considérer que la capacité qui correspond à activer, désactiver, construire un réseau informel entretient une forme de culture diffuse de changement.

La communication du changement vécu sur le terrain, par les acteurs présentent deux caractéristiques : la permanence et l'imprévisibilité. En ce qui concerne la permanence nous voulons signifier qu'il y a un processus continu de micro changements dans le quotidien. Cependant, en même temps que ce processus est continu, il est également dans son actualisation imprévisible, non programmable et non pilotable de l'extérieur. Il procède d'une logique non linéaire. Enfin, nous souhaitons questionner l'ensemble de ces comportements qui relèvent également, selon nous, d'un savoir tacite.

\section{Le changement par proaction : émergences et processus continu}

49 L'anticipation par l'action des salariés, la proaction est très fréquente dans l'organisation. Nous considérons qu'il y a proaction à chaque fois que l'action précède tout réajustement procédural éventuel. Une telle proaction comporte au niveau cognitif une forme de préaction c'est-à-dire une forme d'anticipation. Les proactions qui nous intéressent sont celles, nombreuses, qui contiennent une part de micro-changement, par exemple, d'une part lorsqu'il y a un écart vis-àvis des routines procédurales et, d'autre part lorsqu'il y a une innovation dans un vide procédural. Dans tous les cas, cette capacité à proagir renvoie à une forme d'expertise ordinaire : on va par exemple agir différemment avec tel ou tel client interne ou externe en fonction de différentes variables qui sont saisies en temps réel et de manière indicielle, on va raccourcir des délais imposés par les circuits formels en usant des réseaux relationnels informels, on va solutionner un problème technique toujours par le réseau informel, etc.

Il peut s'agir d'éviter certains micro dysfonctionnements, mais aussi de répondre par anticipation aux attentes ou aux objections des clients internes et externes, d'appliquer concrètement des procédures hiérarchiques insuffisamment spécifiques, d'intégrer concrètement les NTIC dans des tâches spécifiques, etc. Afin d'illustrer ce propos, nous allons citer quelques résultats empruntés à l'une des études de cas que nous avons réalisées ces dernières années. Il s'agissait d'étudier l'intégration des NTIC dans la communication d'accueil (Bernard, 1992). Si le personnel a été formé à la microinformatique il n'a quasiment jamais été formé à l'intégration du micro dans l'entretien commercial ou dans l'entretien d'accueil. Une telle intégration relève des initiatives locales des acteurs, en l'occurrence de leurs capacités à changer les modalités de la conduite d'entretien. Par exemple, dans des fonctions d'accueil dans des sociétés de 
service (agences de voyage, micro-informatique, etc.) mais aussi dans des collectivités locales, les employés en contact avec les clients ou les usagers conduisent différemment l'entretien selon les clients et selon la nécessité de consulter plus ou moins longtemps l'écran informatique de leur poste de travail. Des stratégies de communication émergent à cette occasion. Par exemple, nous avons pu observer que, dans certains cas, des interactions impliquant sous une forme anthropomorphique et conative l'ordinateur comme partenaire virtuel entraient dans ces stratégies de communication en temps réel. Dans d'autres cas, on pouvait observer au contraire une forme d'effacement, de neutralisation de la présence du micro par une non verbalisation quant au recours à l'outil technologique. Il était en quelque sorte absent de la dimension verbale de l'interaction. Dans d'autres cas enfin, il était omniprésent dans la dimension non verbale de l'entretien (critères: contact visuel, durée de consultation/durée entretien) ce qui conduisait l'employé à nouer une interaction verbale et non verbale minimale avec le client.

51 Nous pourrions multiplier les exemples qui montrent que, dans l'organisation, les opportunités quotidiennes de changement et d'innovation sont pléthores pour les salariés, il s'agit bien d'émergences dans un processus continu. Cependant malgré leur caractère " aléatoire ", tous ces comportements correspondent à un savoir et à un savoir faire tacite, à des compétences que nous allons essayer de mieux saisir.

\section{Les communicants de peu ou le plus vieux canal du monde}

52 Toutes les formes de micro coopération par le bas qui permettent d'atteindre les objectifs professionnels, mais aussi l'écart entre les prescriptions et l'actualisation du changement tel que les acteurs le font vivre dans la quotidienneté, révèlent selon nous un savoir et un savoir faire tacite. Le propos peut sembler banal, il l'est moins si l'on tente d'en saisir le processus.

Tout d'abord, nous pouvons souligner qu'il s'agit d'une micro communication invisible ou opaque pour les spécialistes de la communication qui sont en fait des spécialistes de la communication formalisée et de la communication médiatisée ${ }^{4}$. Cette opacité peut être comprise, paradoxalement, parce que les canaux activés sont à la fois les mieux partagés, les plus intimes et les plus individualisés, ceux qui impliquent le corps: voix, geste, mimique et regard. Les frontières traditionnelles de la communication organisationnelle excluraient en somme le soma communicationnel. L'opacité est également liée au caractère peu approprié de la méthode déductive, dominante en communication formalisée, pour saisir des phénomènes qui procèdent d'une autre logique et qui requièrent donc une autre méthode.

54 Afin d'approcher les processus complexes de cette micro communication, il nous a semblé important d'accepter pour présupposé que chaque acteur est un communicateur, « un communicant de peu ». Par un détournement sémantique, nous suggérons d'adapter la notion anthropologique "de gens de peu " (Sansot, 1991) à la situation de tout acteurcommunicant dans l'organisation qui est inévitablement en situation de communication et donc qui participe, modestement certes, mais effectivement cependant et expérentiellement à des formes de coproduction, de co-construction du contexte dans un certain espace communicationnel ${ }^{5}$. L'adverbe modestement s'entend dans trois acceptions : à petite échelle, c'est-à-dire dans le sens d'un espace communicationnel réduit, mais aussi avec modestie, et enfin en activant le canal le plus modeste, celui que 
nous nommons le plus vieux canal du monde, le canal corporel. Celui-ci relève en fait d'une multicanalité : le verbal, le vocal et le gestuel.

À canal modeste, causes et effets complexes. Nous allons tenter de saisir quelque peu une telle complexité.

\section{Sens commun}

Tout d'abord, la notion de communicants de peu, peut être éclairée par une approche par le sens commun. En effet, le sens commun en action produit des grilles d'intelligibilité à partir d'un processus largement indiciel. Ces grilles non explicitées contribuent par exemple à guider l'action professionnelle dans une démarche de réajustement permanent. Il représente une forme de métissage culturel. Le sens commun est un lieu d'acculturation en ce qui concerne la culture communicationnelle en ce sens que la pratique de la micro communication impose implicitement une réflexion concernant l'altérité, l'identité, la médiation. Il est aussi un lieu de résistance « au tout managérial et au tout libéral » car s'y s'expriment, en particulier, d'autres formes symboliques du lien social ${ }^{6}$. Notre hypothèse est que ce sens commun se forme et se déforme selon des processus complexes dans lesquels interviennent cependant aussi des formes de médiation identifiables.

Est-il utile de rappeler que le sens commun est également un objet d'étude légitime pour les sciences et techniques cognitives? En particulier les thèmes de la valorisation du contexte et du sens commun (Bogdan, 1988) qui concourent à la définition du concept d'enaction croisent nos préoccupations. Pour ces sciences, la cognition vivante est la capacité à poser des questions pertinentes qui surgissent à chaque moment de l'action quotidienne (Varela, 1989). Autrement dit, selon ces recherches le monde se réalise plutôt qu'il est pré défini. La connaissance ne serait plus un miroir de la nature, c'est la fin de la représentation (voir Heidegger, Merleau Ponty, Foulcault). "Le contexte et le sens commun ne sont pas des artefacts résiduels pouvant être éliminés grâce à des règles plus sophistiquées, ils sont en fait l'essence même de la cognition créatrice $~{ }^{7}$. La communication ne se présente plus comme le transfert d'information d'un émetteur vers un destinataire mais comme «le modelage mutuel d'un monde commun au moyen d'une action conjuguée : notre réalisation par les actes de langage prête vie à notre monde » (Varela, 1989).

Le sens commun communicationnel en organisation est donc un objet d'étude pertinent, selon nous, afin de saisir comment des communicants de peu, par ailleurs acteurs économiques, sujets et citoyens, communiquent à partir de grilles d'intelligibilité non explicitées. Nous formulons deux hypothèses :

59 - premièrement que le sens commun en action procède d'une logique inductive,

60 - deuxièmement que l'étude du sens commun procède d'une méthode inductive.

\section{Énoncé total}

61 La notion de communicants de peu est susceptible selon nous de rendre compte de la coproduction communicationnelle, informelle, quotidienne, continue, ordinaire qui participe anonymement à la coproduction du sens dans le contexte de l'organisation. Le communicant de peu, sujet psychosomatique et néanmoins expert de la communication ordinaire, produit des énoncés qui peuvent être appréhendés à partir de la notion 
d'énoncé total. Cet énoncé total de la communication ordinaire se compose donc d'une dimension verbale et d'une dimension non verbale impliquant la subjectivité (Orecchioni, 1980) et plus encore l'intersubjectivité.

En ce qui concerne l'énonciation, tout consultant ou chercheur ayant côtoyé le terrain s'est heurté à cette difficulté qui consiste à décoder, dans un contexte donné, lors d'un travail de recueil de données par exemple, la multiplicité des métaphorisations, des analogisations (Morgan, 1989; Veran, 1992), bref toutes les formes de surcodage (Sfez, 1973,1993) qui renvoient à une multiplicité de regards, de micro cultures. Un tel parcours est une véritable heuristique de l'induction pour le chercheur : l'expérience sensorielle de chacun est confrontée à des schémas croisés de réception - interprétation, dans une perspective dialogique, qui conduisent à une co-construction co-textuelle et contextuelle. Nous avons été confrontés à une telle difficulté en tant que chercheur par exemple dans l'analyse de la réception par des salariés de la notion de changement, changement annoncé par la direction (Bernard et Durampart, 1996). Nous avons pu mesurer que la communication officielle descendante de changement était ré-interprété par les salariés selon des règles métacommunicationnelles implicites et diffuses. Une telle réinterprétation produisait des résultats différents en terme d'adhésion donc en terme politique. Ces règles renvoyant à un savoir communicationnel tacite issu de l'expérience des uns et des autres et de l'histoire communicationnelle de l'organisation telle qu'elle a été vécue par les acteurs. De plus la notion de multi-contexte semblait s'imposer puisque le surcodage s'effectue également en fonction d'une pluriappartenance à plusieurs contextes de la sphère publique ou privée (l'équipe, l'organisation, le métier, l'appartenance syndicale, la famille politique, la famille, etc.)

Au-delà ou en-deçà de l'analyse des résultats de l'énonciation, plus difficile encore pour un chercheur est la traduction de la dimension non verbale de la communication. Il y a en effet une difficulté méthodologique majeure qui est «qu'il n'existe pas de langage codé qui permette de transcrire un mouvement en protocole écrit comme il en existe un pour transcrire la parole » (Frey et alii, 1984) ${ }^{8}$. Une telle difficulté est généralement résolue par l'oubli et la méconnaissance, en quelque sorte un acte manqué méthodologique. Au « flot du comportement non verbal» (Mahl, 1968) ${ }^{9}$ répond la sécheresse et l'aridité du comportement d'étude. Compte tenu d'un tel obstacle méthodologique, nous pouvons en conclure que, dans tous les cas où il y a recueil d'énoncés autres qu'écrit, les données recueillies sont au mieux une interprétation de données fragmentaires et partielles. Il s'agit d'une herméneutique portant sur des fragments. Autrement dit la notion même de donnée mérite d'être questionnée au regard des multiples limites méthodologiques et ce, indépendamment de la rigueur de chercheurs dont on sait qu'ils maîtrisent les méthodes inductives de l'étude empirique en science sociales.

Un tel oubli lorsqu'on se fixe pour objectif d'intégrer à l'orchestre de la communication organisationnelle le soma de la communication ré-émerge du creux au plein. Autrement dit, la réussite, implicitement admise, de l'acte manqué méthodologique émerge en dévoilant le refoulé. Bien évidemment mettre en évidence la dimension somatique ne nous fait pas oublier la complexité des interactions cognitivo-psycho-somatiques.

Bref, le chercheur est soumis à un véritable casse-tête inductif, que nous avons tendance à résoudre en ce qui nous concerne par le recours à une logique abductive. C'est à dire en quelque sorte un va et vient entre les cas, les résultats d'une part et, d'autre part les règles, celle-ci sont en fait des méta, des inter-règles issues d'une tentative de construction interdisciplinaire, d'un métissage culturel. 

dimension non langagière et une dimension paralangagière. La non langagière relève d'une "sémiotique du non verbal », la paralangagière relève de catégories co-textuelles les deux concourants à un énoncé total composé des trois sous systèmes : verbal, vocal et gestuel (Cosnier, Brossard, 1984). On pourrait ajouter que l'énoncé total intègre la relation émetteur-récepteur-contexte (Shanon, 1983). Bien évidemment, en complément, d'autres études analysent les invariants de la NVC: les signes non verbaux (Scherer, 1984), le regard réciproque » (Cook, 1984), les mouvements faciaux (Ekman et Friesen, 1984), etc.

69 Nous avons eu l'occasion d'expérimenter, dans des études de cas la pertinence d'un tel apport scientifique, par exemple en observant comment, à l'occasion du déroulement d'un événement de changement, le coup d'envoi d'un projet interétablissements, la sémiosis non verbale, en particulier la proxémie et la sémiotique des parcours, révélait ce que la stratégie verbale d'énonciation des uns et des autres tentait de dissimuler en terme d'alliances de changement. Dans le cas présenté, la sémiosis non verbale était manifestement en décalage avec le texte.

En conclusion, mettre en évidence l'influence de la communication des communicants de peu dans les situations de changement mais aussi dans les situations professionnelles de manière plus générale soulève doublement la question de la pertinence de l'induction :

71 - par l'objet d'études, celui-ci semble révéler des mécanismes internes que l'on peut décrire par l'induction,

par la méthode, les chercheurs sont conduits à résoudre de nouveaux problèmes en termes méthodologiques; une des voies se trouve incontestablement du côté de l'approche inductive.

\section{Nœud des rationalités et enjeux scientifiques} - Il y a une actualité de la communication informelle qui émerge des discours de modernité du changement.

- Or, un retour sur la littérature dévoile l'accumulation d'études sur le thème de l'informel, c'est-à-dire une histoire de cette notion.

En d'autres termes, Tout se passe donc comme si une forme de cryptomnésie frappait la discipline. Une autre manière d'aborder cette question est de constater un décalage entre le savoir accumulé dans la littérature sur la nécessité de l'informel et la faible légitimité récurrente de celui-ci. Au-delà on observe que des approches, des concepts sont exhumés avec le parfum de l'invention après parfois quasiment un de-mi-sècle d'oubli.

Communication et organisation, 12 | 1997 
77 Cette cryptomnésie associée au constat de la difficulté de penser l'articulation entre formel et informel autrement que techniquement pour ne pas dire anecdotiquement posent problème. Nous y percevons une difficulté récurrente à penser l'articulation de logiques différentes, de rationalités différentes. C'est ce que nous nommons l'impensée de la communication organisationnelle, son point aveugle.

Or, nous prétendons que la communication organisationnelle en tant que discipline ne peut faire l'économie d'une réflexion sur ce que nous nommons, de manière un peu caricaturale il est vrai, le nœud des rationalités. Une telle réflexion est par ailleurs en cours de défrichement, sur le terrain où, " par le bas ", les questions de logiques et de rationalités sont également posées.

\section{Les discours de modernité du changement et la légitimation de la communication de changement informelle}

79 Aux changements réels s'ajoutent donc des discours de plus en plus nombreux sur la prescription du changement. La nécessité de changement est mise en énoncés, énoncés fréquemment performatifs.

On peut distinguer deux périodes dans ce processus d'énonciation :

81 - dans un premier temps, le changement a été théorisé (années 80),

82 - dans un deuxième temps, le changement a été opérationnalisé, technicisé (à partir du début des années 90).

83 En ce qui concerne la théorisation, la pensée du changement organisationnel dans la littérature française est souvent associée à la pragmatique de la communication de l'Ecole de Palo Alto. Les apports théoriques retenus des auteurs du Collège invisible sont principalement :

84 - la distinction entre contenu et processus de changement (Bartoli, 1990),

85 - l'approche paradoxale du changement, par exemple «plus c'est la même chose, plus ça change » (Thévenet, 1988),

86 - la distinction entre changement de niveau 1 et changement de niveau 2.

87 Un tel éclairage théorique légitime la communication comme discipline pertinente afin de comprendre et d'accompagner le changement, il a été très socialisé, via les consultants et une certaine littérature technique, auprès de l'encadrement. Par exemple, de nombreux praticiens considèrent que lorsqu'un changement échoue c'est fréquemment parce que le processus d'accompagnement du changement, c'est-à-dire la communication de changement, a été négligé.

Cette légitimation se fait, nous l'avons dit, par la pragmatique c'est-à-dire dans le cadre $\mathrm{du}$ paradigme interactionniste. Cependant, qu'on ne s'y trompe pas, l'interactionnisme est largement dominé par un présupposé majeur qui lui est déterministe et évolutionniste comme nous l'avons déjà souligné.

Implicitement dans nombre de discours, le changement est associé à l'idée de survie, de telle sorte qu'il est impossible, du point de vue du sens commun de ne pas y adhérer. Il y aurait une forme de déterminisme, d'univocité autour de la question du changement que nous avons déjà eu l'occasion de souligner. Ce présupposé renvoie à la tradition positiviste du changement social, dans la lignée d'Auguste Comte et d'Herbert Spencer, où le changement social est présenté comme un processus universel d'évolution. Le 
changement des organisations serait donc une des formes du changement social. Le changement provoqué par les évolutions techniques serait donc un processus linéaire, "positif». Ainsi la technique devient un déterminisme qui exclurait toute autre alternative. De ce point de vue la technique est pensée comme exogène à la culture, elle s'imposerait comme pression externe incontournable. Un tel postulat est évidemment éminemment discutable. En particulier on peut lui opposer que la technique dans sa pensée et dans ses réalisations est chargée de culture. Les thèmes du changement et de la culture sont d'ailleurs associés dans la littérature en organisation. Tantôt la culture d'entreprise est présentée comme un frein au changement tantôt elle est présentée comme le cadre de référence incontournable pour tout changement.

Donc tout se passe comme si la théorisation avec la mise en avant du paradigme interactionniste n'effaçait pas la prégnance des présupposés positivistes.

91 Dans un deuxième temps, avec les années 90, le changement est opérationnalisé, rationalisé. En presque vingt ans, nous sommes ainsi passés de l'idée de changement à une normalisation $d u$ changement organisationnel. Concrètement, l'ensemble des organisations quelqu'en soient leur secteur d'activité, leur forme juridique, leur appartenance au secteur privé ou au secteur public, est concerné par le changement. Par voie de conséquence, le chantier du «changement» est devenu l'un des chantiers alimentant les interventions de la consultance en communication. Les interventions sont centrées sur le passage de l'organisation fonctionnelle à une organisation des processus avec une forte rationalisation et avec la construction d'un ensemble de référentiels.

En résumé par la reconfiguration organisationnelle, on assiste à un double mouvement d'externalisation économique/ d'internalisation culturelle. On observe ainsi, au niveau économique, un mouvement d'externalisation maximale des coûts financiers auquel répond au niveau culturel, pour les acteurs des organisations, un mouvement d'internalisation des valeurs libérales. Celui-ci a été étudié en psychologie sociale (Dubois, 1994). En ce qui concerne ce mouvement d'internalisation, on pourrait dire que l'on passe d'une régulation à une autorégulation. Le desserrement de la traditionnelle relation d'autorité ainsi provoqué est accompagné d'un resserrement par la mise en procédures. À $\mathrm{y}$ regarder de près, les valeurs explicites d'autonomie qui sont mises en avant à cette occasion s'inscrivent dans des règles implicites de méta-hétéronomie de l'autonomie. Autrement dit, on assiste à une dématérialisation, une dé-interaction du contrôle qui ne se joue plus dans l'interaction mais dans auto conformité aux procédures et dans auto évaluation. De manière complémentaire, certains auteurs observent que « la production frugale », issue du "dégraissage », c'est-à-dire du licenciement massif, s'accompagne "d'une internalisation au sein de l'entreprise de l'idéologie de la guerre économique » (Crozier, Seriyex, 1994).

93 Autant dire que l'idéologie de la communication change de cap : la guerre économique n'est plus conjugable avec le management participatif. D'autant plus que certaines conséquences du reengineering, en particulier les plans sociaux, déconsidèrent de facto une décennie de discours et d'expériences du management participatif. Cependant, la contradiction entre reengineering et management participatif n'est peut-être qu'apparente. En effet, les théories d'internalité nous conduisent à voir dans le management participatif une étape, sous la forme d'un apprentissage, vers l'internalisation de normes libérales.

Le changement serait donc moderne, et la communication informelle serait légitimée. 


\section{Légitimation de la communication informelle}

$\mathrm{Si}$, de manière générale et historique, la communication informelle peut être définie comme la dimension oubliée de la communication managériale, on note, depuis le début des années 80 , l'émergence d'un discours nettement plus favorable à l'informel. Nous allons explorer le passage de ce silence à un début de légitimation. Pour cela un retour en arrière est nécessaire.

Donc, traditionnellement, l'informel embarrasse les directions et l'encadrement qui ont préféré pendant longtemps méconnaître cette dimension communicationnelle en se réfugiant dans une approche à la fois formalisée et ritualisée de la communication. En ce qui concerne la formalisation, il s'est agi par exemple de privilégier le canal papier et en ce qui concerne le canal oral il s'est agi d'en ritualiser les formes: les réunions et les entretiens formels en sont un bon exemple. Autant de tentatives pour «cadencer le moteur humain » (Mattelart, 1994), autant d'actualisation de la métaphore mécaniciste de la communication.

Concrètement, l'informel était en effet fréquemment doublement réduit :

- premièrement réduit aux phénomènes de rumeur eux-mêmes traduits en terme d'entropie ou de déficit de communication formelle,

- deuxièmement réduit à une variable de convivialité des cultures d'équipe.

En d'autre terme, l'informel c'était la rumeur ou la machine à café.

On peut aussi analyser une telle tendance comme participant d'un syndrome plus général, celui de la mise en procédures des organisations comme condition de leur permanence. Faut-il rappeler que des travaux de référence datant de 35 ans avaient déjà souligné l'existence, positive pour l'action des organisations formelles, d'une tension entre la nécessité de changer et celle de maintenir un certain état de leur structure par la production de procédures de plus en plus spécialisées (Blau et Scott, 1962). L'informel est bien situé plutôt du côté du changement et le procédural plutôt du côté de la permanence.

Cependant, dans la dynamique de changement managérial repérable depuis le début des années 80 , les cadres, en particulier ceux de proximité, n'hésitent plus à se former à des modèles de communication sensés les aider à " gérer l'informel ». Le déclencheur d'un tel mouvement est à trouver du côté des discours du management participatif où il était recommandé aux cadres de devenir des animateurs, des coordinateurs; bref de développer des compétences de cadre de proximité. Un tel discours est particulièrement présent en ce qui concerne le management des programmes qualité par les cercles de progrès et les cercles de qualité. D'une certaine manière, la structure en équipe de projet a prolongé cette tendance puisque le chef de projet est aussi un animateur d'équipe.

3 Une telle reconnaissance de l'informel n'est pas univoque. À y regarder de près, les séminaires en communication proposés aux cadres dans leur projet de gestion de l'informel produisent inévitablement des effets de normalisation (Bernard, 1996).

En ce qui concerne ces séminaires, on peut considérer que les objectifs managériaux sont de deux types. Premièrement, ils consistent à normaliser l'intersubjectivité, le consensuel, à gérer la communication directe, de face à face. Le moyen mis en œuvre est une diffusion de discours qui relèvent de prés ou de loin de la pragmatique de la communication. Deuxièmement, ils consistent à convaincre l'encadrement qu'il a la 
responsabilité de la production du sens et proposent une normalisation des pratiques discursives (Bautier, 1994). Dans les deux cas, on peut observer une forme de "bricolage " culturel. Les normes sont diffusés sous forme de modèles fermés qui dissimulent mal de très nombreuses approximations théoriques.

Dans les deux cas, il y a une volonté de normalisation de la communication informelle, voire de normalisation des processus de subjectivation. Cette dernière dimension souligne la confirmation d'une rupture entre une conception de l'individu au travail acteur et une conception de l'individu au travail sujet ${ }^{10}$. Quoiqu'il en soit, compte tenu de l'importance de cette tendance, on peut considérer qu'il y a désormais une certaine culture communicationnelle diffuse dans les organisations. D'autant plus que, dans les années 80 , ces séminaires joints au discours ambiant sur «l'explosion de la communication" ont servi à socialiser un des axiomes centraux de Palo Alto à savoir "qu'on ne peut pas ne pas communiquer».

Désormais, dans les énoncés managériaux, desserrer le formel c'est plutôt favoriser le changement et l'innovation ${ }^{11}$, il y aurait aussi une modernité de l'informel. Bien évidemment l'émergence discursive de l'informel s'inscrit également dans de nouvelles approches stratégiques de la ressource humaine. Avec le remplacement de la productivité par la compétitivité, la ressource humaine est décrite comme ressource stratégique (Thierry, 1994). Par ailleurs, cette valorisation de l'informel n'est pas étrangère non plus à la montée des formes individualisées de la gestion des relations sociales.

107 À la légitimation managériale, s'ajoute une légitimation scientifique. Les chercheurs spécialisés en analyse stratégique et en analyse de changement soulignent également qu'il est indispensable de la prendre en compte et qu'il faudrait même en valider la légitimité (Pichault, 1994). Quant à nous, nous avons souligné l'importance de la communication informelle dans les échanges "culturels scientifiques et techniques" (Bernard-Peyrolle, 1994). Par ailleurs, toujours au niveau des énoncés scientifiques, des propositions de «conceptions alternatives de l'organisation» (Veran, 1992) centrées autour de la métaphore organiciste de l'organisation attribue à l'interaction une place parfois centrale dans l'architecture organisationnelle en termes de «tissu relationnel » ou de « tissu d'interdépendances $»^{12}$.

108 Il y a donc convergence entre des énoncés managériaux et des énoncés scientifiques pour valider une approche communicationnelle renouvelée de l'organisation dans laquelle l'informel aurait une certaine légitimité.

109 Or, cette actualité de l'informel semble faire fi d'une littérature «historique » somme toute abondante sur le thème.

\section{Un exemple de cryptomnésie : la critique du modèle bureaucratique centralisé, formel}

110 L'histoire des idées managériales révèle une forme singulière de cryptomnésie, en ce sens que des textes oubliés réémergent régulièrement avec des arguments de modernité. Il en est ainsi pour ce que nous avons nommé «l'éternel retour de l'école des relations humaines » (Bernard, 1996). Certains théoriciens des organisations, dans la filiation des études conduites par les chercheurs de Harvard fondateurs du courant des relations humaines, ont défini l'entreprise " comme système vivant » où l'informel a été analysé d'abord comme une compensation au système formel (Mayo, 1933) puis comme une des 
dimensions à part entière, un sous système interagissant du système sociotechnique avec les recherche du Tavistock Institute of Human Relations (Emery et Trist, 1969).

Plus généralement, la littérature sociologique dressant la critique du modèle bureaucratique centralisé, formel apporte une précieuse contribution à l'analyse de l'informel. Le sujet est assez souvent abordé par les questions de la motivation. Par exemple, nous avons eu l'occasion de souligner comment la théorie de la motivation de Maslow, datant des années 40 , avait trouvé une nouvelle jeunesse en ayant été réutilisée comme argument de pertinence du management participatif et plus précisément comme argument en faveur d'une implication des salariés dans les cercles de qualité et les cercles de progrès dans les années 80, (Bernard-Peyrolle, 1990).

Qu'on ne s'y trompe pas cependant, légitimation récente et connaissances redécouvertes n'ont que peu fragilisé le modèle formel. Celui-ci se nourrit de «l'effet sociétal » français qui régénère de manière latente le modèle industriel de "bureaucratie mécaniciste " (Maurice, Sellier et Silvestre, 1982).

113 Ainsi donc, le chemin du changement n'est pas linéaire : il y a des avancées et des reculs, des voies sans issue, des sentiers abandonnés et redécouverts, des autoroutes qui bifurquent, etc. Bref, en toute légitimité, chercheurs et praticiens se demandent quelles sont les rationalités à l'œuvre.

\section{Parcours vers une inter-multirationalité}

114 Parmi les présupposés du changement, l'un d'entre eux et non le moindre est celui de la rationalité (Pichault, 1993). le changement est rationnel, le changement doit produire plus de rationalité, ce qui se traduit sur le terrain par une volonté de rationalisation. Or, le paradigme de la rationalité en économie et en micro-économie a une histoire. En même temps qu'il domine, ce paradigme a été violemment mis en cause par la brèche ouverte avec l'invention du concept de «rationalité limitée » (Simon, 1979). Elle est limitée en particulier parce que celui qui est en situation de décision ne peut maîtriser l'ensemble des informations dont il aurait besoin pour délibérer.

115 Dans cette brèche le paradigme de la rationalité a été bousculé par des tentatives d'importation de théories diverses. Par exemple la théorie du chaos qui a produit des théorisations nouvelles « le modèle de la boîte à ordures » (March et Olsen, 1991), mais aussi des modèles de management diffusés sous le terme de «chaos management ». Il a également été bousculé par le paradigme interactionniste qui a été étendu à toutes les composantes de l'organisation avec l'approche du « tout acteur » : l'individu, les objets, les construits sociaux et culturels, seraient des acteurs en interaction. Le retour de l'acteur annoncé par les sociologues (Touraine, 1984) a connu un succès dépassant toute prévision.

116 En organisation, tous ces apports pourraient nourrir la thèse de la multirationalité (Sfez, 1986) dans le sens où la notion de rationalité limitée ${ }^{13}$ de toute situation de décision pourrait être enrichie par la mise en perspective d'autres rationalités toutes aussi limitées, les rationalités communicationnelles, voire "l'intrusion de l'irrationalité " (Sfez, 1986). En d'autres termes la multirationalité serait poly-limitée.

117 Or le débat relatif au nœud des rationalités est un débat essentiel d'une actualité critique à la fois scientifiquement et pratiquement. En effet, il est implicite ou peut-être méta, dans les questions centrales de l'apprentissage organisationnel ${ }^{14}$ et de la légitimation des 
savoirs, en particulier du savoir tacite (Reix, 1995). Il est essentiel dans la question du changement. Celle-ci est rabattue sur la question des techniques et des méthodes procédurales, ce qui en soit est déjà une question, insuffisante certes, mais une question néanmoins de rationalité. Au-delà la question de: «quelle modernité pour l'organisation? » inclut la question de quelles rationalités. Celle-ci est souvent traduite en terme de polarité : norme-contingence, matérialité-immatérialité, etc.

Dans ce cadre, la NVC, mériterait, selon nous, d'être reconnue comme objet de recherche en communication organisationnelle, avec le même statut de modernité que les NTIC. Une telle légitimité scientifique serait à relier à la légitimité déjà acquise en anthropologie de la communication (Winkin, 1981, 1994). Nous proposons également de relier ces deux champs, le champ de la NVC et celui des NTIC. Nous avons conscience que notre proposition peut être entendue comme la défense d'un «monisme » communicationnel, c'est-à-dire en l'occurrence d'un continuum communicationnel de l'univers mental à l'univers matériel des $\mathrm{NTIC}^{15}$ mais aussi d'un continuum contexte-émetteur-récepteur. L'hypothèse mérite d'être mise en discussion selon nous. Notre hypothèse étant par ailleurs qu'il est nécessaire de fonder théoriquement une définition élargie et donc interdisciplinaire de l'homo communicans ${ }^{16}$ afin d'apporter une contribution pertinente au socle épistémologique de la communication organisationnelle. Dans notre proposition la notion d'homo communicans serait posée en distinction à l'homo socius-oeconomicus, un peu dans la perspective d'un rapprochement entre la définition que donne Wiener de l'homo communicans (Wiener, 1948,1952) et la définition que donne Ghiglione de l'homme communiquant (Ghiglione, 1986). Autrement dit la porosité de la communication organisationnelle où tout communique ne peut être saisie que dans une dynamique d'ouverture des territoires scientifiques, dans un rapprochement entre « digitalisation » et " analogisation » (Watzlawick, 1972).

119 Le projet même des défenseurs du reengineering qui engagent à renoncer au modèle fonctionnel pour se placer au plus près des processus réels de l'activité réelle valide, en quelque sorte malgré lui, l'activité communicationnelle des communicants de peu. C'est un rappel de la définition de l'individu comme "système informel » (Bougnoux, 1991) ${ }^{17}$ nous ajouterons «complexe». Or, ce système informel n'est, selon nous, que partiellement "encastré dans l'économique $»^{18}$. Une telle caractéristique d'une part légitime une définition anthropologique de ce paradigme, et, d'autre part de ce fait offre à la communication managériale un élargissement de son cadre de référence car celle-ci, est enchâssée dans l'économique avec la domination du paradigme du marketing (Floris, 1996). Autrement dit, la nouvelle ambition communicationnelle mais aussi sociétale de nombreuses organisations passe aussi selon nous par la légitimation d'une approche anthropologique de la communication organisationnelle. Une telle légitimation validerait une forme d'importation et d'exportation d'une culture communicationnelle par les acteurs-communicants de peu. On pourrait parler de processus de communi« $\mathrm{s}$ »ation globale, dans le sens où tout salarié participe activement à la construction communicationnelle de l'organisation, dans le sens où la mise en communication globale de l'organisation passe aussi par la connexion des mises en communication de chacun.

En conclusion, les chercheurs en anthropologie de la communication organisationnelle pourraient se fixer pour objectif une tentative de rapprochement de l'ensemble des contributions scientifiques pertinentes pour appréhender la communication organisationnelle dans une perspective métacommunicationnelle. Bien évidemment ne pourrait être omise une analyse des rapports de pouvoir qui caractérisent le jeu des 
contributions communicationnelles en organisation. Ce qui nous conduit aussi à la pensée médiologique de la communication lorsqu'il est dit que les pensées gagnantes, sont des actes d'organisation (Bougnoux, 1991, Debray, 1991), nous ajouterons y compris en communication. Ce qui revient aussi à réaffirmer l'originalité de la communication organisationnelle comme amplification de la caractéristique des SIC «reliant /.../ l'élaboration théorique aux mondes professionnels » (Miège, 1995) ${ }^{19}$.

Nous préconisons en substance la mise en place d'un programme de recherche en anthropologie de la communication organisationnelle. Dans un tel programme une attention toute particulière pourrait être accordée à l'induction, à la fois comme méthodologie mais aussi comme paradigme à articuler avec celui de "l'abduction" (Peirce, 1933) dans la perspective peircéenne des trois étapes dans le processus «de recherche de la vérité " (Deledalle, 1990) ${ }^{20}$. Dans une telle perspective, il nous semble important de préciser que la connaissance de l'induction, en tant qu'objet, que logique et que méthode, ne peut progresser que si le chercheur accepte que sa compétence inductive, faite de rigueur méthodologique, soit aussi pré-façonnée par des règles culturelles, c'est-à-dire des présupposés issus d'une autre logique : la logique déductive. Ainsi la condition communicationnelle du chercheur serait rabattue sur celle de tout communicant de peu.

ADAM G. \& REYNAUD J.D., Conflits du travail et changements social, Paris PUF, 1978.

BARDELLI P., L'impact de l'introduction des nouvelles technologies dans l'usine hétérogène, Annales du management, Paris, Economica, 1992.

BARTOLI A., Communication et organisation, Paris, Ed. d'Organisation, 1990.

BAUDRY B., L'économie des relations interentreprises, Paris, Repères-La Découverte, 1995. organisationnelle de la SFS1C, Rennes, juin 1996, à paraittre.

BERNARD PEYROLLE F, Contribution de la Culture scientifique et technique à la modernité de l'entreprise, in Rasse P., Giordan A., Girault Y., Culture scientifique et technique de l'entreprise, Z'éditions, 1994, pp. 136-146.

BERNARD PEYROLLE $\mathrm{F}$, «Les séminaires de communication destinés à l'encadrement, variété des formes, uniformité des présupposés ", Analyse de 20 séminaires, WP, Université de Nice-Sophia Antipolis, n 10,1990-93.

BERNARD-PEYROLLE F, «L'intégration des NTIC dans la communication d'accueil », étude de cas n ${ }^{\circ}$ 7, Université de Nice-Sophia Antipolis, 1992.

BERNARD-PEYROLLE $\mathrm{F}$, « La communication des programmes qualité, » étude de cas $\mathrm{n}^{\circ} 3$, Université de Nice-Sophia Antipolis, 1990.

BERNARD-PEYROLLE F, « Quand additionner, c'est multiplier, Logiques de communication interne et Programmes qualité », Humanisme et entreprise, n 190,1991. 
BLOCH LAINE F., Pour une réforme de l'entreprise, Paris, Le Seuil, 1963.

BOGDAN R.J., "Attitudes mentales et psychologie du sens commun ", Hermès 3, Paris, 1988, pp. 56-84.

BOGDAN R.J., "Sens commun et science cognitive", Sfez L. (sous la direction de) Dictionnaire de la communication, Paris, PUF, 1993, tome 1, pp. 907-912.

BOUGNOUX D., La communication par la bande, Paris, Ed. La découverte, 1991.

BOUGNOUX D., Sciences de l'information et de la communication, Paris, Larousse, 1993.

BOURDIEU P., Réponses

BRETON P., L'utopie de la communication, Paris, La Découverte, 1992.

BROUSSEAU E., L'économie des contrats, technologies de l'information et coordination interentreprises, Economie en Liberté, Paris, PUF, 1993.

CHEVALIER F, «Les cercles de qualité à bout de souffle? ", Annales des Mines, Gérer et comprendre, $n^{\circ} 7$, juin 1987.

CIORAT B., Penser à l'envers, Paris, C. bourgeois, 1991.

COHENDET P., LLERENA P., Flexibilité, information et décision, Paris, Economica, 1989.

CROZIER M. \& SERIEYX H., (sous la direction de), Du management panique à l'entreprise du XXIe siècle, Boulogne, Maxima Laurent éditeur, 1994.

CROZIER M., «Ce que nous a apporté Herbert Simon », RFG, n 94, juin-juil.-août 1993, pp. 85-86.

DELEDALLE G., « Abduction », Dictionnaire de philosophie, Les notions philosophiques, Paris, PUF, tome 1,1990 .

DUBOIS N., La norme d'internalité et le libéralisme, Grenoble, PUG, 1994.

EMERY F.E. et TRIST E.L., "Socio-technical systems », Emery systems thinking, Penguin, 1969.

FLICHY P., L'innovation technique, Paris, La Découverte, 1995.

FLORIS B., La communication managériale, Grenoble, PUG, 1996.

FREY S. et alii, "Analyse intégrée du comportement non verbal et verbal dans la communication ", Cosnier J. et Brossard A., La communication non verbale, Ed. Delachaux Niestlé, 1984, pp. 145-227.

GILLES P., Crises et cycles économiques, Paris, Armand Colin, Cursus, 1996.

GRENARD A. et alii, "Normalisation et organisation de l'industrie-Approches pluridisciplinaires », Paris, Revue d'Economie Industrielle, $\mathrm{n}^{\circ}$ spécial, Ed. Techniques et économiques, $\mathrm{n}^{\circ}$ 75, 1996.

HAMMER M., CHAMPY J., Reengineering (trad franc.), Paris, Dunod, 1993.

Hermès 3, Psychologie ordinaire et sciences cognitives, 1988.

HUARD P., « Rationalité et identité : vers une alternative à la théorie de la décision dans les organisations ", Revue économique, n 3, 1980, pp. 541-565.

JACOB G., « Le reengineering de l'entreprise », Hermès, Paris, 1994.

KAPFERER Jean Noël, Rumeurs, Paris, Le Seuil, 1987.

KARCHER X., "Maîtrise des flux de produits-Maîtrise des flux d'informations », Revue Française de Gestion industrielle, $\mathrm{n}^{\circ} 1,1988$. 
KOENIG G., «L'apprentissage organisationnel : repérage des lieux », RFG, n 97 , janv.-fév. 1994, pp. 76-83.

LE MOËNNE C., «Communication «by smiling around » et crise managériale », Réseaux, $\mathrm{n}$ ○64, mars-avril 1994, pp. 29-52.

LEMOIGNE J.L., Le constructivisme, Paris, ESF, tome 1 : des fondements, 1994, tome 2 : des épistémologies, 1995

LEVY P., "Vers une nouvelle économie du savoir ", Pour une nouvelle économie du savoir, Solaris, Rennes, PUR, nº 1, 1994, pp. 113-134.

LINHART D., « Le taylorisme n'est pas mort », Cahiers de l'ANVIE, n 38, avril 1994.

LIU M., «Les nouvelles logiques en organisation du travail », Le Nouvel Economiste-FNEGE, numéro spécial : Nouvelles logiques de production, 1988.

MARCH J.G., OLSEN J.P., « Le modèle du « Carbage Can » dans les anarchies organisées », in

MARCH J.G (sous la direction de), Décisions et organisations, Paris, Ed. d'Organisation, 1991.

MATTELART A., L'invention de la communication, Paris, Ed. La Découverte, 1994.

MAURICE M., SELLIER F, SIVESTRE J.J., Politique d'éducation et organisation industrielle en France et en Allemagne, Paris, PUF, 1982.

MAYO E., 77k ; human problems of an industrial civilization, New York, Macmillan, 1933.

MIÈGE B., La pensée communicationnelle, Grenoble, PUG, 1995.

MIEGE B., La société conquise par la communication, Grenoble, PUG, 1989.

MILGRAM M., «Les formalismes du hasard », L'auto-organisation, DUMOUCHEL P., DUPUY J. P. (sous la direction de), Paris, Le Seuil, 1983, pp. 187-210.

MOLES A., Théorie structurale de la communication et société, Paris, Masson, 1986.

MORGAN G., Images de l'organisation, Les presses universitaires de Laval, Ed. Eska, 1989.

OLLIVIER B., L'acteur et le sujet, Paris, Desclée de Brouwer, 1995.

PARAPORANIS C., "Une approche intégrée de la production en flux tendus ", Revue Française de Gestion industrielle, $\mathrm{n}^{\circ}$ 4,1990.

PICHAULT F, Ressources humaines et changement stratégique, Bruxelles, DeBoeck Université, 1993.

PIERCE C.S., Collected Papers, Cambridge Mass., The Belknap Press of Harvard University Press, 1933.

POLANYI K., La grande transformation: aux origines politiques et économiques de notre temps, Paris, Gallimard, 1983.

POPPER K.R., « La démarcation entre la science et la métaphysique », in JACOB P. ( sous la direction de), De Vienne à Cambridge, Paris, Gallimard, 1980, pp. 131-192.

REIX R., « Savoir tacite et savoir formalisé dans l'entreprise », RFG, n 105 , sept.-oct. 1995, pp. 17-29.

SCHIEB P. A., «La communication inter-entreprises informelle ou micro-coopération », Humanisme et Entreprise, 1986, nº 156.

SFEZ L., Critique de la communication, Paris, Le Seuil, 1988. 
SFEZ L., Critique de la décision, Presses de la Fondation Nationale des Sciences Politiques, 1973, $4^{\circ}$ éd. 1992.

SFEZ L., La décision, PUF, QSJ, n 2181, 2éd. 1988.

SFEZ L., "Le surcode dans les organisations", Dictionnaire de la communication, PUF, tome 1, 1993, pp. 694-702.

SFEZ L., «Multirationalité dans les organisations », Dictionnaire de la communication, PUF, 1993, tome 1, pp. 625-639.

SHANON B., "Que disent les oiseaux, réflexions sur une théorie de la communication ", L'auto-organisation, de la physique à la politique, Dumouchel P. et Dupuy J.P ( sous la dir de), colloque de Cerisy, Éd. du Seuil, 1983, pp. 407-411.

SIMON H., "Rational Decision making in Business Organizations », American Economic Review, sept 1979, pp. 493-513.

SUDREAU P., La réforme de l'entreprise, Rapport du comité d'experts, Paris, UGE, $10 / 18,1975$.

THEPOT J., «La rationalité limitée ou l'arlésienne de la théorie économique », RFG, n 94, juin-juil.-août 1993, pp. 79-84.

THEVENET M., «Plus c'est la même chose, plus ça change », RFG, n 68 , mars-avril-mai 1988, pp. 89-92.

THIERRY D., la place réelle des ressources humaines dans les stratégies d'entreprise ", RFG, n 97, janv.-fév. 1994, pp. 43-48.

TOURAINE A., Le retour de l'acteur, Paris, Fayard, 1984.

VARELA F.J., Connaître les sciences cognitives, Paris, Seuil, 1989.

VERAN L., « Diagnostics et représentations de l'organisation ». Annales du management, Economica, 1992, pp. 793-807.

VERLEY P., La révolution industrielle, Paris, Gallimard, Folio histoire, 1997.

WATZLAWICK P. et alii, Une logique de la communication, Paris, Seuil, 1972.

WIENER N., Cybernétique et société, Paris, Deux-rives, 1952.

WINKIN Y., Anthropologie de la communication, de la théorie au terrain, Bruxelles, De Boeck Université, 1996.

WINKIN Y, "Communication interpersonnelle et interculturelle», SFEZ L. (sous la direction de) Dictionnaire de la communication, Paris, PUF, 1993, tome 1, pp. 413-516.

WINKIN Y, La nouvelle communication, Paris, Ed. du seuil, 1981.

WOMARCK J.P., JONES D.T., ROOS D., Le système qui va changer le monde, Paris, Dunod, 1992.

WOOD S, «Le modèle japonais : postfordisme ou japonisation du fordisme?, Vers un nouveau système productif ? », Durand J.P., Syros, 1993. 


\section{NOTES}

1. Jacob G, U reengineenng de l'entreprise, Hermès, Paris, 1994, p. 23.

2. Nous détournons l'expression proposée par Patrice Flichy - «tout bouge ensemble »- y compris de son contexte -l'innovation technique-Flichy, 1995.

3. Cf. ci-dessous : "La communication du changement vécu : non linéarité »

4. Une telle observation est compatible selon nous avec le constat par ailleurs d'une certaine réhabilitation du corps dans l'entreprise depuis les années 70 . Celle-ci est corrélable a plusieurs éléments que nous citons en vrac: le mouvement plus général de libération du corps, la polysensualité publicitaire, les projets et les recettes d'influence et de manipulation en tous genres, les progrès de l'ergonomie, etc.

5. La notion d'espace communicationnel s'inspire de la notion d'espace social forgée par Bourdieu.

6. Ces autres formes relèvent de la société civile, de la religion, de la famille

7. Varela, 1989, p. 98.

8. Frey et alii, 1984, p. 146.

9. Mahl G.F., 1968, cité par Frey et alii p. 147.

10. La définition de sujet est à trouver dans le rapport entre des sujets, cf. à ce propos : Ollivier, 1995.

11. Voir a ce sujet par exemple, «La dynamique humaine du changement ", Progrès du management. $\mathrm{n}^{\circ} 19$, février 1995.

12. Veran, 1992, p. 803.

13. Des auteurs soulignent que si le concept de rationalité limitée a été fécond intellectuellement, les économistes ne s'en sont pas véritablement emparés pour renouveler leur analyse en particulier de la décision; $c f$. Thépot $\mathrm{J}$, « La rationalité limitée ou l'artésienne de la théorie économique ", RFG, n 94, juin-juil.-août 1993, pp. 79-84. Michel Crozier de son côté souligne que Herbert Simon a été un des «pères spirituels» de la nouvelle sociologie qui s'élabore depuis 20 ans dans la réflexion croisant culturel et pouvoir, Crozier M., «Ce que nous a apporté Herbert Simon », RFG, n 94, juin-juil.-août 1993, pp. 85-86.

14. Gérard Koenig souligne les deux versions des études dans ce domaine qui sont d'une part la gestion de l'expérience accumulée et d'autre part l'intelligence de l'expérimentation ; Koenig G, «L'apprentissage organisationnel : repérage des lieux ", RFG, n 97, janv.-fév 1994, pp. 76-83

15. Bateson défend un point de vue moniste, anticartésien, lorsqu'il indique que «le discours scientifique n'a pas à établir de distinction entre l'univers matériel et l'univers mental ", in Vers une écologie de l'esprit, débat résumé par Yves Winkin (1993. P. 432).

16. La métaphore cybernétique nous offre l'image d'une communication sans sujet. Wiener a le premier proposé la notion d'homo communicans, dans son esprit il s'agissait d'une réponse à la barbarie contemporaine par la constitution d'un nouveau lien social fondé sur la transprence et le rôle des « machines à communiquer ».

17. «/.../il y a du donné, de l'implicite ou du milieu qui précède, borde et contient notre capacité de calcul », Bougnoux, 1991, p. 105.

18. L'expression est de Karl Polyani, elle est citée par Michel Beaud, in Le Monde, Les grands entretiens du Monde, numéro spécial de Dossiers et documents du Monde, juin 1996, p. 73.

19. Miège, 1995, p. 110.

20. «L'abduction suggère les hypothèses ou idées générales que la déduction développe et que l'induction met a l'épreuve et partant vérifie ou falsifie », Deledalle, 1990, p. 4. 


\section{AUTEUR}

FRANÇOISE BERNARD

Université de Provence, IUP ICIS 